\title{
COMMUNICATION
}

\section{Périostite ossifiante de la mandibule. A propos d'un cas pédiatrique.}

\author{
Taïhi Nassif I1,2, Radoï L1,3 \\ 1. Faculté de Chirurgie Dentaire, Université Paris V - Paris Descartes \\ 2. Servie d'Odontologie, Hôpital Charles Foix - Ivry-sur-Seine \\ 3. Servie d'Odontologie, Hôpital Louis Mourier - Colombes
}

\section{Introduction}

La périostite proliférative (PP), appelée également périostite est une pathologie osseuse bénigne chronique nonsuppurative. Elle fait partie des ostéomyélites chroniques et se manifeste par une prolifération et une ossification du périoste en regard d'une inflammation ou d'une infection osseuse chronique à germes de faible virulence (1). La PP touche plus fréquemment les os de la main et les os longs des enfants et des adolescents. La sphère cranio-faciale est rarement concernée, avec une atteinte préférentielle de la mandibule. L'étiologie est souvent dentaire (parodontale, endodontique, péricoronaire). Néanmoins, des formes idiopathiques de PP sont décrites (1). Elles représentent un défi diagnostique.

\section{Observation}

Un enfant de 9 ans, en bonne santé générale, était adressé par sa pédodontiste pour une tuméfaction génienne basse gauche évoluant depuis 3 mois, sans cause dentaire apparente et ne répondant pas au traitement antibiotique (amoxicilline et métronidazole). Le patient était apyrétique et ne décrivait aucune douleur. L'examen endobuccal montrait une voussure corticale vestibulaire en regard des dents 34,35 et 36, recouverte d'une muqueuse d'aspect normal. Aucune anomalie n'était retrouvée à l'examen dentaire et parodontal. L'orthopantomogramme montrait des images radioclaires infra-centimétriques isolées dans les régions apicales prémolo- molaires mandibulaires gauches, associées à une image faiblement radio-opaque, fusiforme, accolée au bord inférieur de l'hémi-mandibule gauche. Le scanner mandibulaire montrait un épaississement périosté de l'hémi-mandibule gauche $(4 \mathrm{~cm}$ de diamètre en vestibulaire et $1 \mathrm{~cm}$ de diamètre en lingual). II confirmait la présence des lésions radio-claires dans l'os médullaire et des perforations corticales mandibulaires, communicant avec des zones lacunaires au sein de la réaction périostée. L'imagerie par résonnance magnétique écartait toute atteinte des tissus mous. Le bilan biologique était normal. La biopsie de la lésion montrait un aspect histologique compatible avec une PP. En l'absence de toute cause évidente, la forme idiopathique de la maladie a été retenue. L'antibiothérapie initiale a été prolongée de 3 mois. Une régression progressive de la tuméfaction a été notée pendant le suivi. Au contrôle de 18 mois, la symétrie faciale était rétablie et la cicatrisation osseuse était complète.

\section{Discussion}

La PP est une pathologie osseuse bénigne dont l'étiologie est souvent patente et l'aspect radiologique caractéristique : apposition de lamelles osseuses néoformées, parallèlement à la corticale osseuse qui est préservée (en « pelure d'oignon »). Le cas rapporté représentait un défi diagnostique du fait de l'absence d'une cause évidente et de l'aspect radiologique atypique (perforation des corticales et nécrose de l'os néoformé entre le périoste et la mandibule), signe d'une lésion plus agressive (2,3). Dans le cas où l'étiologie dentaire est trouvée, le traitement ou l'extraction de la dent, avec ou sans antibiothérapie associée, conduit à la guérison de la PP. Dans ce cas idiopathique, seulement un traitement non chirurgical a été réalisé.

\section{Conclusion}

Ce cas montre la difficulté diagnostique des périostites chez l'enfant, en absence de cause dentaire patente et en présence de modifications radiologiques atypiques. Ainsi, il est important de considérer la PP parmi les diagnostics différentiels de nombreuses pathologies bénignes et malignes, de cause locale ou générale, responsables de périostites.

insene.taihi@parisdescartes.fr

(C) The authors, published by EDP Sciences. This is an Open Access article distributed under the terms of the Creative Commons Attribution License 4.0 (http://creativecommons.org/licenses/by/4.0/). 